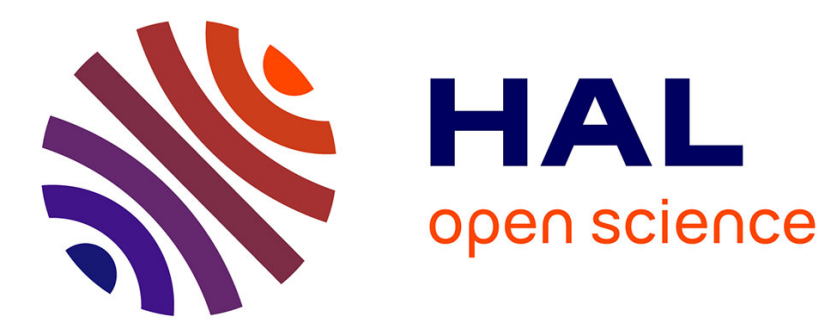

\title{
MHD simulation of electric currents in the solar atmosphere caused by photospheric plasma motion
}

\author{
J. C. Santos, J. Büchner
}

\section{To cite this version:}

J. C. Santos, J. Büchner. MHD simulation of electric currents in the solar atmosphere caused by photospheric plasma motion. Astrophysics and Space Sciences Transactions, 2007, 3 (1), pp.29-33. hal-00297487

\section{HAL Id: hal-00297487 \\ https://hal.science/hal-00297487}

Submitted on 4 Dec 2007

HAL is a multi-disciplinary open access archive for the deposit and dissemination of scientific research documents, whether they are published or not. The documents may come from teaching and research institutions in France or abroad, or from public or private research centers.
L'archive ouverte pluridisciplinaire HAL, est destinée au dépôt et à la diffusion de documents scientifiques de niveau recherche, publiés ou non, émanant des établissements d'enseignement et de recherche français ou étrangers, des laboratoires publics ou privés. 


\title{
MHD simulation of electric currents in the solar atmosphere caused by photospheric plasma motion
}

\author{
J. C. Santos and J. Büchner \\ Max-Planck Institute for Solar System Research, Katlenburg-Lindau, Germany \\ Received: 8 August 2007 - Revised: 15 October 2007 - Accepted: 15 October 2007 - Published: 4 December 2007
}

\begin{abstract}
Photospheric plasma motion may cause the buildup of electric currents in the solar atmosphere. These electric currents may then dissipate and heat the corona, cause EUV and X-ray Bright Points (BPs) and trigger flares. In this work we use a 'data driven' 3D MHD model to study the generation of electric currents in the solar atmosphere by photospheric plasma motion. The model was applied to photospheric measurements below an observed EUV-BP. The observed photospheric magnetic field and the derived horizontal plasma motion were used as initial and boundary conditions of a simulation. The horizontal motion gave rise to electric currents in the chromosphere, transition region and lower corona. The currents formed in the area where the motion was applied and above the main concentrations of the photospheric magnetic field.
\end{abstract}

\section{Introduction}

For more than six decades a key question has puzzled researchers: what is the mechanism of solar coronal heating? At the moment the attempts to answer this question are contained in two main groups of heating models: wave (AC) heating and electric current (DC) heating. The source of energy for both mechanisms is the photospheric energy reservoir. AC heating (Alfvén, 1947) requires that the magnetic field is moved around in the solar photosphere faster than the Alfvén crossing time. Waves are generated in this process which must then be dissipated in the solar corona to generate heat. DC heating requires plasma motion in the photosphere at timescales longer than the Alfvén crossing time. Such relatively slow motion can generate electric currents. These currents cannot be easily dissipated through conventional Joule heating (Parker, 1972), but by collisionless anomalous resis-

Correspondence to: J. C. Santos

(santos@mps.mpg.de) tivity (Büchner and Daughton, 2006; Büchner and Elkina, 2005, 2006). The anomalous resistivity is assumed to be dependent of the velocity of the current carrier (Roussev et al., 2002) and acts only in the points where the velocity of the current carrier exceed the thermal velocity of the electrons.

EUV and X-ray BPs are a directly observable phenomena of the heating of the solar corona. The main properties of BPs are summarized in, e.g. Madjarska et al. (2003) (and references therein): BPs are small scale features, $30-40$ arcsec wide, of enhanced X-ray and EUV emission. Their coronal structure corresponds to miniature loops evolving on a time scale of approximately $6 \mathrm{~min}$. The average life time of a BP is $20 \mathrm{~h}$ in EUV and $8 \mathrm{~h}$ in X-ray observations. BPs temperatures vary between $1.3-1.7 \cdot 10^{6} \mathrm{~K}$. Their densities are $2-4$ times higher than the coronal average $\left(5 \cdot 10^{14} \mathrm{~m}^{-3}\right)$. However, one of the most important characteristics that can help to understand their nature is that they are associated to moving, vertical and horizontal motions, bipolar magnetic features.

Models developed to explain the observational features associated to BPs consider the interaction between the magnetic field of the moving bipolar magnetic feature and the surrounding magnetic field (Priest et al., 1994; Parnell et al., 1994; Longcope, 1998; Rekowski et al., 2006). However, they do not consider the role of the plasma moving through regions of strongly diverging magnetic flux. Büchner et al. (2004a,b) and Büchner (2006) showed that horizontal plasma motion in the photosphere and chromosphere causes the formation of a localized current sheet in and above the transition region at the position of the EUV-BP. They claim that the enhanced current flow can make the current sheet resistive and allows stress relaxation by current dissipation and reconnection which power the BP.

An open question is that of the location of electric currents in the solar atmosphere, which cannot be observed directly. For this sake simulations are necessary. In this work we use a 'data driven' 3D MHD model (Büchner et al., 2005) to study

Published by Copernicus Publications on behalf of the Arbeitsgemeinschaft Extraterrestrische Forschung e.V. 
the formation of electric currents in a BP region observed on 19 January 2006 (M. Madjarska, private communication). We derived the velocity field responsible for the evolution of the photospheric magnetic features between 16:00 UT and 16:30 UT, on that day, and applied it as boundary condition to the model. The model and the observational data are described in Sect. 2 and Sect. 3. The results obtained for the parallel and perpendicular currents are presented in Sect. 4. A brief discussion of the results and some conclusions are given in Sect. 5.

\section{Model description}

The MHD model solves the complete set of MHD equations

$$
\begin{aligned}
\frac{\partial \rho}{\partial t} & =-\nabla \cdot \rho \boldsymbol{u} \\
\frac{\partial \rho \boldsymbol{u}}{\partial t} & =-\nabla \cdot \rho \boldsymbol{u} \boldsymbol{u}-\nabla p+\boldsymbol{j} \times \boldsymbol{B}-v \rho\left(\boldsymbol{u}-\boldsymbol{u}_{0}\right) \\
\frac{\partial \boldsymbol{B}}{\partial t} & =\nabla \times(\boldsymbol{u} \times \boldsymbol{B}-\eta \boldsymbol{j}) \\
\frac{\partial p}{\partial t} & =-\nabla \cdot p \boldsymbol{u}-(\gamma-1) p \boldsymbol{\nabla} \cdot \boldsymbol{u}+(\gamma-1) \eta j^{2}
\end{aligned}
$$

together with Ohm's law, Ampère's law and the equation of state

$$
\begin{aligned}
\boldsymbol{E} & =-\boldsymbol{u} \times \boldsymbol{B}+\eta \boldsymbol{j} \\
\nabla \times \boldsymbol{B} & =\mu_{0} \boldsymbol{j} \\
p & =2 n \kappa_{B} T .
\end{aligned}
$$

where $\rho$ is the plasma density, $\boldsymbol{u}$ is the plasma velocity, $\boldsymbol{B}$ is the magnetic field, $p$ is the thermal pressure and $\mathrm{T}$ is the plasma temperature. The quantity $\boldsymbol{u}_{0}$ denotes the velocity of neutral gas, to which the plasma is coupled, at least in the chromosphere and photosphere.

In the momentum equation, the collision term is used to describe the transfer of momentum from a neutral gas to the plasma. There, $v$ represents the collision frequency between the neutral gas and the plasma. In the photosphere and chromosphere the collision frequency is large compared to the inverse Alfvén time. This allows the use of a value for this frequency, that is bigger than the inverse Alfvén time. As a consequence, in these regions the plasma motion is strongly coupled to the neutral gas. In the solar corona the density is much smaller so that the coupling between plasma and neutral gas vanishes.

The initial magnetic field configuration is obtained from the photospheric line of sight (LOS) magnetic field using a force-free extrapolation $(\boldsymbol{\nabla} \times \boldsymbol{B}=\alpha \boldsymbol{B})$. The extrapolation method is described in Otto et al. (2007). It is similar to that developed in Seehafer (1978) but takes in account that the boundary conditions in the extrapolation must be consistent with the boundary conditions applied to the MHD model. The initial magnetic field is assumed to be potential $(\boldsymbol{\nabla} \times \boldsymbol{B}=0)$ and after the relaxation phase it assumes a nonforce-free form, where currents along the magnetic field and perpendicular to it are created. The initial density mimic the height stratification observed in the solar atmosphere. The gravity force is not considered in the model and the plasma is assumed to be in hydrostatic equilibrium $(p=c t e)$ at the beginning of the simulation. The temperature profile is then computed from the equation of state (Eq. 7).

When the plasma and magnetic field are put together in the simulation box, the system is not anymore in equilibrium. For this reason, at the beginning of the simulation, a relaxation phase is necessary to bring the system closer to an equilibrium situation. During the relaxation the system is allowed to evolve and after some time all motion due to unbalanced forces is dissipated, i.e. a 'force equilibrium' state is reached. We now apply to the relaxed equilibrium a photospheric horizontal motion to simulate the resulting evolution of the system. The velocity in the lower boundary is imposed by the neutral gas velocity. The plasma is dragged behind since it interacts with the neutral gas by collisions (momentum equation). The motion is generated by a combination of three velocity vortices. This way we reproduce the velocity pattern calculated from the evolution of the photospheric magnetic field structures. The neutral gas velocity must satisfy $\boldsymbol{\nabla} \cdot \boldsymbol{u}_{0}=0$ in order to inhibit a piling up of plasma and magnetic field.

\section{Data and methodology}

We applied the model to an EUV-BP detected on 19 January 2006. To obtain the velocity responsible for the evolution of the photospheric magnetic structures associated to the BP we used the local correlation tracking (LCT) technique (November and Simon, 1988). First, the LOS component of the photospheric magnetic field was filtered using a Fourier filter to select the first eight modes of interest. Then, LCT was applied to the filtered magnetograms separated by a time interval of approximately $30 \mathrm{~min}$, covering the interval between 16:00 UT and 16:30 UT. As a result we obtained a velocity pattern, which is shown in Fig. 1. The formally obtained horizontal motion around the positive magnetic field concentration was, however, an effect of the emergence of magnetic flux in that region interpreted by LCT as a horizontal velocity pattern (Démoulin and Berger, 2003). For this reason, we focused the simulation on the horizontal motion derived from the displacement around the negative magnetic field concentration. In the simulation model this motion was approximated by using a combination of vortices of velocity (Fig. 2). The initial three-dimensional magnetic field was obtained from a potential extrapolation of the filtered LOS photospheric magnetogram obtained at 16:00 UT using the algorithm described in Otto et al. (2007). 


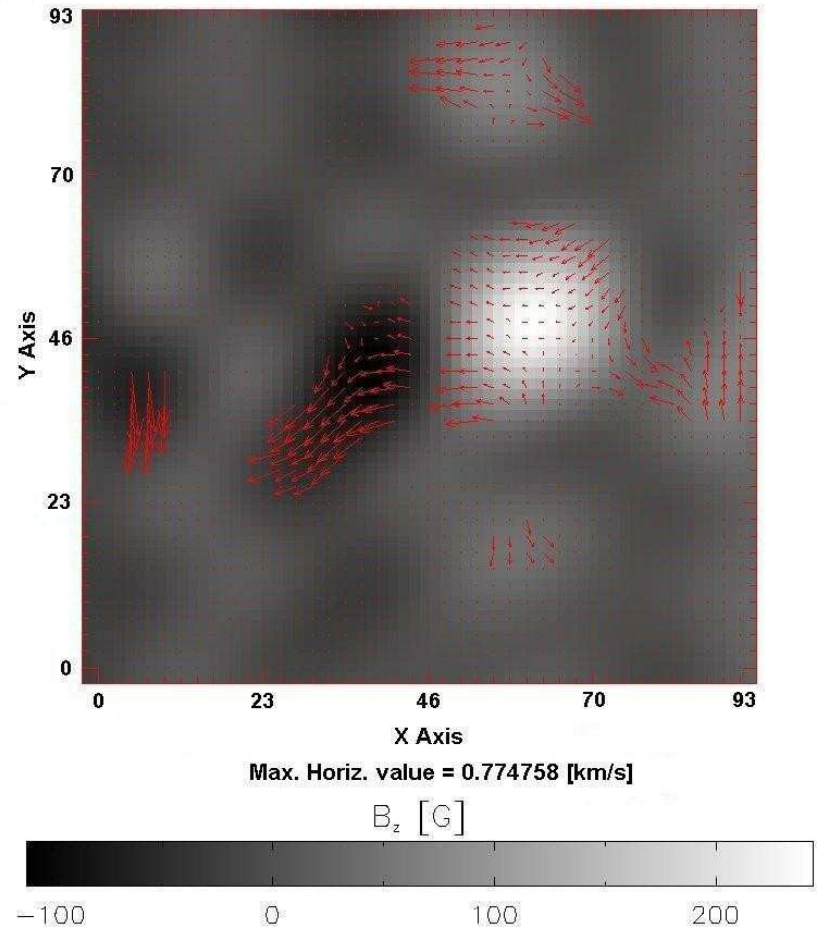

Fig. 1. Horizontal velocity obtained using LCT technique applied to the filtered magnetic field in the interval 16:00 UT - 16:30 UT on Jan $19^{\text {th }} 2006$.

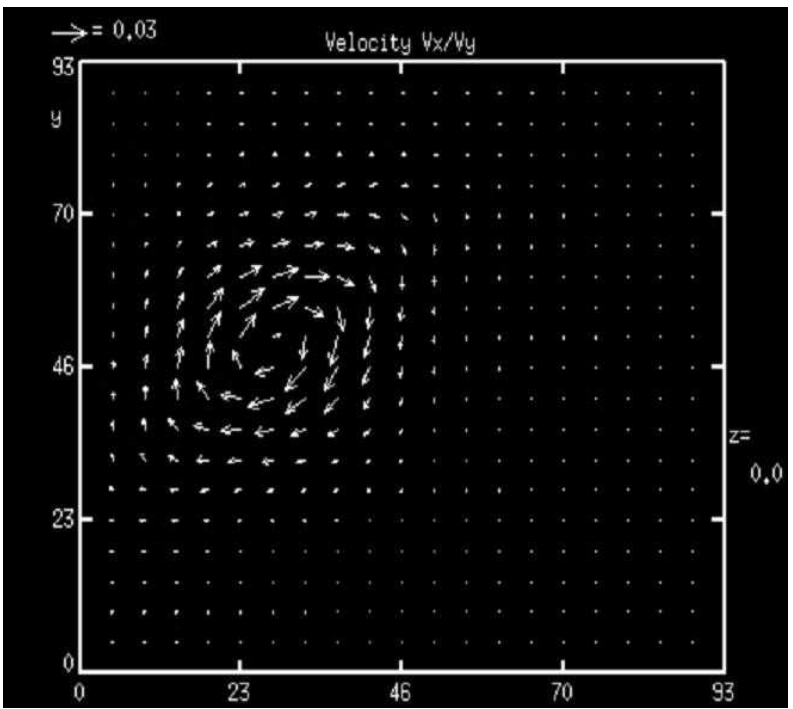

Fig. 2. Horizontal velocity used as boundary condition of the model to approximate the velocity pattern obtained in Fig.1.

\section{Results}

The applied photospheric plasma motion gave rise to electric currents just above the region where it was applied, over
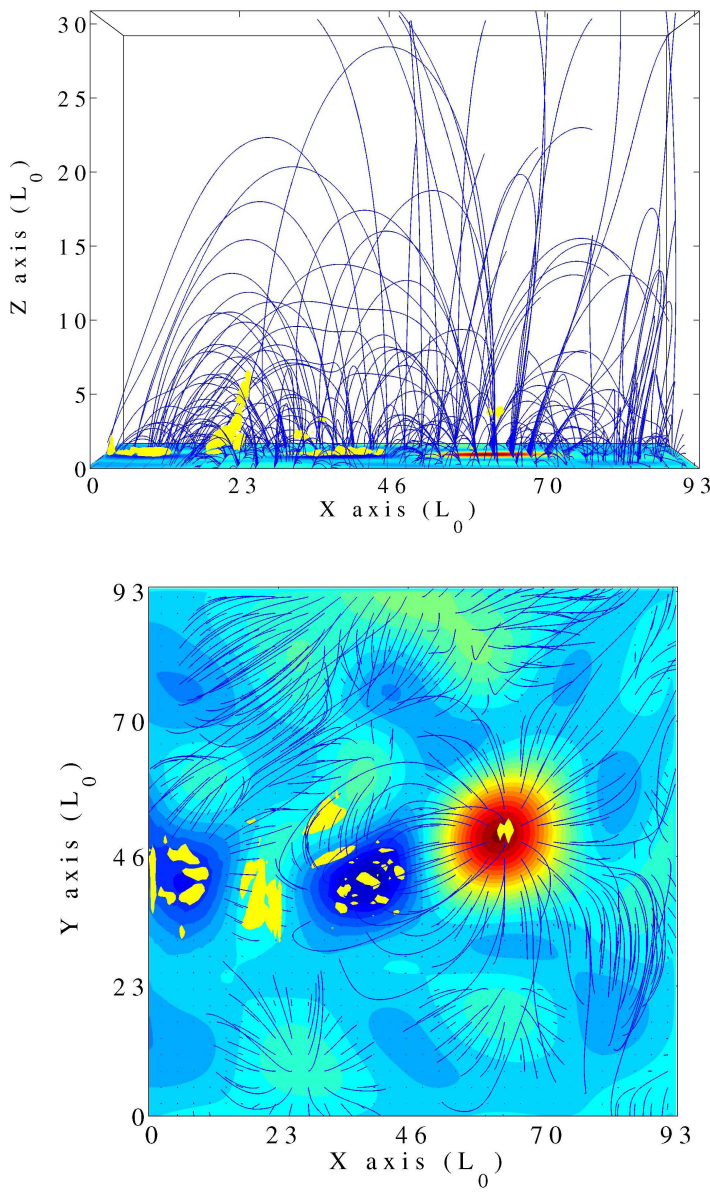

Fig. 3. Lateral view from the negative y-direction (top panel) and top view (bottom panel) of the isosurfaces of a perpendicular current $j_{\perp}=2 j_{0}$, at $t=1300 \mathrm{~s}$. The isosurfaces of perpendicular current are shown in yellow.

the main polarities of the magnetic field. Figure 4 shows the isosurface of a parallel current $j_{\|}=2 j_{0}$, where $j_{0}=\frac{B_{0}}{\mu_{0} L_{0}}$ is the value used to normalize the electric current. This current system results from the application of the velocity pattern of Fig. 2 as a boundary condition to the model. This velocity pattern moves the negative polarity region southward, away from the positive polarity. The motion stretches the magnetic flux tubes, increasing their magnetic energy contents. The horizontal motion also gave rise to electric currents perpendicular to the magnetic field. Figure 3 shows the isosurface of a perpendicular current $j_{\perp}=2 j_{0}$. The parallel and perpendicular currents form just below the solar corona, in the solar atmosphere, and the perpendicular component is less distributed than the parallel component. Note that the currents near the boundary are artifacts of the simulation. The profiles of the current energy

$$
E_{j}(z)=\int_{A} j^{2} d x d y
$$



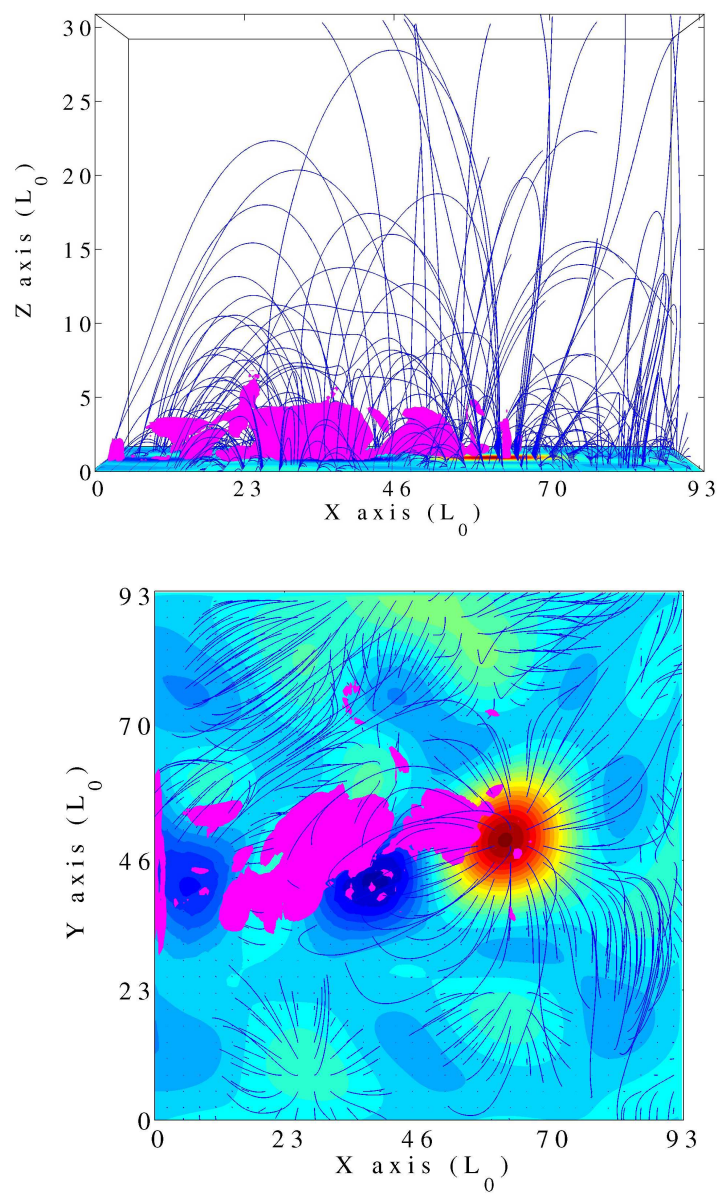

Fig. 4. Lateral view from the negative y-direction (top panel) and top view (bottom panel) of the isosurfaces of a parallel current $j_{\|}=2 j_{0}$, at $t=1300 \mathrm{~s}$. The isosurfaces of parallel current are shown in magenta.

are shown in Fig. 5 for the parallel and the perpendicular currents at $t=1300 \mathrm{~s}$. In this profile the current energy start to increase below $z=15 \mathrm{~L}_{0}$, i.e. near and below the transition region. The energy associated with the parallel current dominates over that associated to the perpendicular current.

We also calculated the evolution in time of the total magnetic energy inside the simulation box

$E_{B}=\int_{V} \frac{B^{2}}{2 \mu_{0}} d V$.

Figure 6 shows the total magnetic energy, in Joules, versus time. It shows that the total magnetic energy increases while the opposite magnetic polarities were moved apart.

\section{Conclusions}

Our simulation has shown that the horizontal motion of the photospheric plasma and magnetic flux gave rise to electric

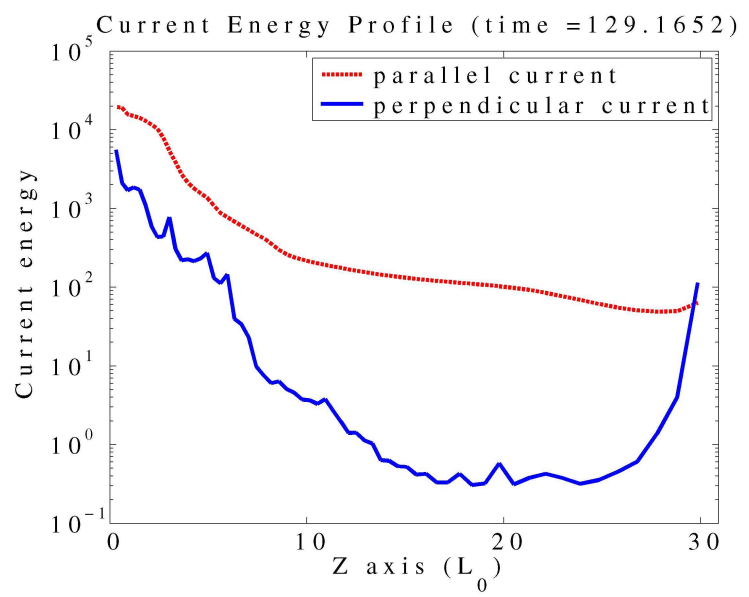

Fig. 5. Height profile of the current energy obtained at $t=1300 \mathrm{~s}$. The height at $z=5$ corresponds to the transition region height.

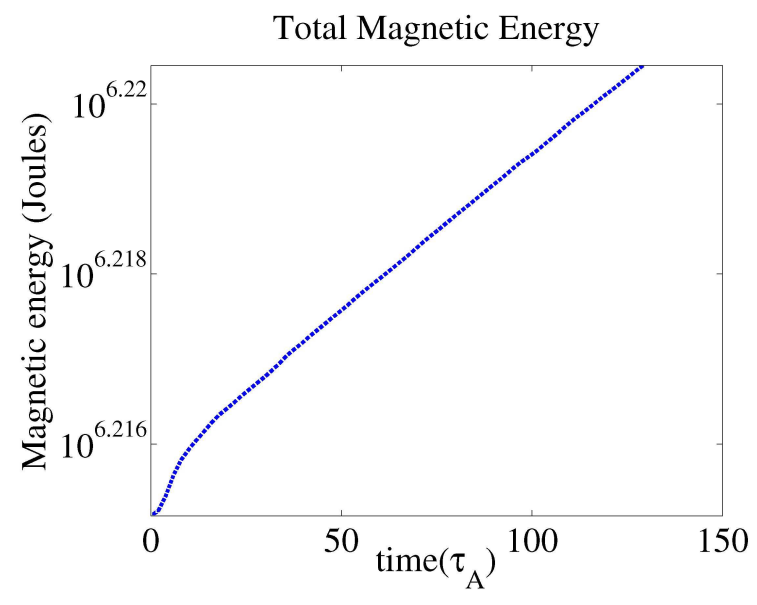

Fig. 6. Total magnetic energy (joules) versus time $\left(\tau_{A}\right)$.

currents mainly in the chromosphere, transition region and lower corona. The currents formed above the region where the motion was applied and above the main concentrations of magnetic field. The component of the electric current parallel to the magnetic field was stronger and spatially more extended than the component perpendicular to the magnetic field. The latter appeared as kernels of current concentration as shown previously in Büchner (2006).

A comparison with TRACE data showed that the area where the current system formed coincides with the area where the EUV-BP appeared (M. Madjarska, private communication). If a temporal sequence of images from trace is analyzed one important characteristic is that indeed the brightening is always distributed approximately in the same area and the kernels of brightening move inside this area. If the mechanism of heating by DC current dissipation would be efficient, as suggested by Büchner and Elkina $(2005,2006)$, 
this current distribution could explain the appearance of the bright point in TRACE data. The fainter extended brightening could be associated to the heating caused by the distributed system of currents parallel to the magnetic field, and the moving brighter kernels would be associated to the more efficient dissipation of the kernels of current perpendicular to the magnetic field (Büchner and Daughton, 2006).

Acknowledgements. J. C. Santos would like to acknowledge the International Max-Planck Research School-IMPRS that supported this work by means of a $\mathrm{PhD}$ fellowship.

Edited by: H.-J. Fahr

Reviewed by: F. Verheest and another anonymous referee

\section{References}

Alfvén, H.: Granulation, magneto-Hydrodynamic waves and the heating of the solar corona, Mon. Not. Roy. Astron. Soc., 107, 211-219, 1947

Büchner, J.: Locating current sheets in the solar corona, Space Sci. Rev., 122, 149-160, 2006.

Büchner, J. and Daughton, W.: Reconnection of Magnetic Fields: Magnetohydrodynamics, Collisionless Theory and Observations, edited by J. Birn and E. R. Priest, Cambridge: Cambridge University Press, 144-153, 2007.

Büchner, J. and Elkina, N.: Vlasov code simulation of anomalous resistivity, Space Sci. Rev., 121, 237-252, 2005.

Büchner, J. and Elkina, N.: Anomalous resistivity of current-driven isothermal plasmas due to phase space structuring, Phys. Plasmas, 13, 2304-2313, 2006.

Büchner, J., Nikutowski, B., and Otto, A.: Magnetic coupling of photosphere and corona: MHD simulation for multi-wavelenght observations, in Multi-Wavelength Investigations of Solar Activity, Proc. IAU Symp., 223, 353-356, 2004.

Büchner, J., Nikutowski, B., and Otto, A.: Coronal heating by tran- sition region reconnection, Proc. SOHO 15 Workshop, Coronal Heating, St. Andrews, Scotland, 6-9 September 2004, ESA SP575, 2004.

Büchner, J., Nikutowski, B., and Otto, A.: Plasma acceleration due to transition region reconnection, Particle acceleration in astrophysical plasmas: Geospace and beyond, edited by D. Gallagher, AGU monograph, Washington, 161-170, 2005.

Démoulin, P. and Berger, M. A.: Magnetic energy and helicity fluxes at the photospheric level, Solar Physics, 215, 203-215, 2003.

Longcope, D. W.: A model for current sheets and reconnection in X-ray bright points, Astrophys. J., 507, 433-442, 1998.

Madjarska, M. S., Doyle, J. G., Teriaca, L., and Banerjee, D.: An EUV bright point as seen by SUMER, CDS, MDI and EIT onboard SoHO, Astron. Astrophys., 398, 775-784, 2003.

November, L. J. and Simon, G. W.: Precise proper-motion measurement of solar granulation, Astrophys. J., 333, 427-442, 1988.

Otto, A., Büchner, J., and Nikutowski, B.: Force-free magnetic field extrapolation for MHD boundary conditions in simulations of the solar atmosphere, Astron. Astrophys., 468, 313-321, 2007.

Parker, E. N.: Topological dissipation and the small-scale fields in turbulent gases, Astrophys. J., 174, 499-510, 1972.

Parnell, C. E., Priest, E. R, and Titov, V. S.: A model for X-ray bright points due to unequal cancelling flux sources, Solar Physics, 153 217-235, 1994.

Priest, E. R., Parnell, C. E., and Martin, S. F.: A converging flux model of an X-ray bright point and an associated canceling magnetic feature, Astrophys. J., 427, 459-474, 1994.

von Rekowski, B., Parnell, C. E., and Priest, E. R.: Solar coronal heating by magnetic cancellation - II. Disconnected and unequal bipoles, Mon. Not. Roy. Astron. Soc., 369, 43-56, 2006.

Roussev, I., Galsgaard, K., and Judge, P. G.: Physical consequences of the inclusion of anomalous resistivity in the dynamics of $2 \mathrm{D}$ magnetic reconnection, Astron. Astrophys., 382, 639-649, 2002.

Seehafer, N.: Determination of constant $\alpha$ force-free solar magnetic fields from magnetograph data, Solar Physics, 58, 215-223, 1978 . 